\title{
Hollow cosmic string: the general-relativistic hollow cylinder
}

\author{
Gérard Clément* \\ Laboratoire de Gravitation et Cosmologie Relativistes \\ Université Pierre et Marie Curie, CNRS/URA769 \\ Tour 22-12, Boîte 142 - 4, place Jussieu, 75252 Paris cedex 05, France \\ Ilhem Zouzou \\ Département de Physique Théorique - Université de Constantine \\ route d'Ain-el-Bey, DZ-25000 Constantine, Algérie
}

June 1, 1994

\begin{abstract}
We determine the different possible space-time metrics inside an infinite rotating hollow cylinder with given energy density and longitudinal and azimuthal stresses, the metric outside the cylinder being chosen of the spinning cosmic string type. The solutions we obtain for various domains of values of the cylinder parameters include a space-time with topologically Euclidean spatial sections, a black-hole solution, a quasi-regular solution, and various wormhole solutions. A solution which is regular only if the longitudinal dimension is compactified might approximately describe spontaneous compactification of the cylinder to a torus.
\end{abstract}

*E-mail: GECL@CCR.JUSSIEU.FR 


\section{Introduction}

The space-time metric at large distance from a thin, straight, infinitely long cosmic string is well known [1, 2] to be flat but non-Minkowskian, with an angular deficit proportional to the linear energy density of the string. On the other hand, the gravitational field in the region of space where the energy-momentum tensor is non-negligible depends on the precise mechanism responsible for the existence of the string. A limiting case which may be of some interest is that of a hollow cosmic string, the energy-momentum tensor being concentrated not on a line but on the surface of an infinitely long cylinder. The gravitational field generated by such a cylinder with given energy density and longitudinal and azimuthal stresses, and rotating about its axis, was in principle determined some time ago by Frehland [3]. One of the results of Frehland's analysis is that, unexpectedly, a rotating hollow cylinder can exist in general relativity only if its energy-momentum is traceless. Actually, as we shall show below, this intriguing result is not general, but follows from the hidden assumption that the apparent singularities of the space-time metric outside and inside the cylinder coincide. Once this unnecessary constraint is removed, Frehland's 'traceless' condition no longer holds.

This remark led us into reanalyzing the problem of the rotating hollow cylinder, along the lines of the analysis carried out in [4 for a rotating closed string in three-dimensional space-time. The space-time metric outside and inside the cylinder is a stationary, cylindrically symmetric solution of the vacuum Einstein equations. We first give, in the second section of this paper, a complete classification of these solutions. We expect that, from a large distance, the hollow cylinder should look like a thin straight cosmic string, so it is natural to choose the metric outside the cylinder to be the well-known conical metric. Making this choice, we apply in the third section the junction method [5] to derive the parameters of the interior metric from the knowledge of the cylinder's energy-momentum tensor, which we assume to have the anisotropic perfect-fluid form. The generic vacuum stationary cylindricallysymmetric metric being singular on a cylindrical surface $S_{-}$coaxial with the matter cylinder, the interior metric we have obtained can be regular in two cases. Either 1) the singular surface $S_{-}$is in the interior region, but the singularity is only a coordinate singularity. This possibility, studied in the

fourth section, includes, along with a solution with global spatial sections 
which are topologically $\mathbf{R}^{3}$ ( $S_{-}$then reduces to the axis of symmetry), two other families of non-topologically Euclidean solutions: black cosmic string solutions, and a family of quasi-regular solutions, including a limiting case which we interpret as corresponding to a closed hollow cosmic string. Or 2 ) the singular cylinder $S_{-}$lies outside the matter cylinder, so that the singularity is only virtual. As discussed in the fifth section, the corresponding solutions can be divided in two classes, according to the fate of test particles falling inside the cylinder, which may either be eventually reflected back to the ouside space, or accelerated towards the cylinder at interior spatial infinity. We close with a brief discussion.

\section{Stationary cylindrically symmetric vacuum space-times}

A stationary cylindrically symmetric metric has three commuting Killing vectors, one of which is time-like. Such a metric can be parametrized in adapted coordinates by

$$
\mathrm{d} s^{2}=\lambda_{a b}(r) \mathrm{d} x^{a} \mathrm{~d} x^{b}-\mathrm{d} r^{2}
$$

$(a, b=0,1,2)$, with $x^{0}=t, x^{1}=\varphi(0 \leq \varphi<2 \pi), x^{2}=z$. With this parametrization, the Einstein equations read [6]

$$
\begin{aligned}
S_{r}^{r} \equiv & \frac{1}{8}\left[\operatorname{Tr}\left(\chi^{2}\right)-(\operatorname{Tr} \chi)^{2}\right]=\kappa T_{r}^{r} \\
S_{b}^{a} \equiv & \frac{1}{2}\left[\chi_{b}^{\prime a}+\frac{1}{2}(\operatorname{Tr} \chi) \chi_{b}^{a}\right. \\
& \left.-\delta_{b}^{a}\left(\operatorname{Tr} \chi^{\prime}+\frac{1}{4}\left[\operatorname{Tr}\left(\chi^{2}\right)+(\operatorname{Tr} \chi)^{2}\right]\right)\right]=\kappa T_{b}^{a}
\end{aligned}
$$

$\left({ }^{\prime}=\mathrm{d} / \mathrm{d} r\right)$, where $\chi$ is the $3 \times 3$ matrix

$$
\chi=\lambda^{-1} \lambda^{\prime}
$$

Surprisingly, although particular solutions of the vacuum equations (2.2) $\left(T_{\nu}^{\mu}=0\right)$ have been previously discussed in the literature [6, 7, 8, no exhaustive classification of these solutions is, to our knowledge, available. In 
vacuum, the equations (2.2) simplify to

$$
\begin{aligned}
& \operatorname{Tr}\left(\chi^{2}\right)=(\operatorname{Tr} \chi)^{2}, \\
& \chi^{\prime}+\frac{1}{2}(\operatorname{Tr} \chi) \chi=0 .
\end{aligned}
$$

Putting

$$
f \equiv \frac{1}{2} \operatorname{Tr} \chi
$$

and tracing eq. (2.5), we obtain the differential equation

$$
f^{\prime}+f^{2}=0
$$

which is solved either by

$$
f=\frac{1}{r-r_{0}}
$$

or by

$$
f=0 \text {. }
$$

In the first, generic case, the full eq. (2.5) is solved by

$$
\chi=\frac{2}{r-r_{0}} A
$$

where $A$ is a constant $3 \times 3$ matrix, which according to eqs. (2.6) and (2.4) is constrained by

$$
\operatorname{Tr} A=\operatorname{Tr} A^{2}=1 .
$$

The integration of eq. (2.3) then leads to

$$
\lambda=C \exp \left[2 A \ln \left|r-r_{0}\right|\right],
$$

where $C$ is a new constant matrix, of signature $(+--)$. The symmetry of the matrix $\lambda$ implies the symmetry relations

$$
C^{T}=C, \quad(C A)^{T}=C A .
$$

The constraints (2.11) are equivalent to the relations

$$
p_{0}+p_{1}+p_{2}=1,{p_{0}}^{2}+{p_{1}}^{2}+{p_{2}}^{2}=1,
$$


between the three eigenvalues of the matrix $A$. If these three eigenvalues are real and different, the matrix $A$ may be brought into the diagonal form

$$
A=\sum_{i} p_{i} n^{(i)} \otimes \bar{n}^{(i)}
$$

where the $n^{(i)}$ are the linearly independent eigenvectors of $A$, and the $\bar{n}^{(i)}$ are the dual basis one-forms such that

$$
\left\langle\bar{n}^{(i)}, n^{(j)}\right\rangle \equiv \bar{n}_{a}^{(i)} n^{(j) a}=\delta_{i j} .
$$

The symmetry properties (2.13) imply that the matrix $C$ is diagonal in the basis $n^{(i)} \otimes n^{(j)}$, so that eq. (2.12) reads

$$
\lambda=\sum_{i} k_{i}\left|r-r_{0}\right|^{2 p_{i}} \bar{n}^{(i)} \otimes \bar{n}^{(i)}
$$

leading to the Kasner-like [9, 10] metric

$$
\mathrm{d} s^{2}=\sum_{i} k_{i}\left|r-r_{0}\right|^{2 p_{i}}\left(\mathrm{~d} y^{i}\right)^{2}-\mathrm{d} r^{2},
$$

where円

$$
\mathrm{d} y^{i}=\bar{n}_{a}^{(i)} \mathrm{d} x^{a} .
$$

If an eigenvalue $p$ is degenerate $(p=0$ or $p=2 / 3)$, then $(A-p)^{3}=$ $(1-3 p)(A-p)^{2}$, so that $(2.12)$ leads to

$$
\begin{aligned}
\lambda= & C\left\{\frac{(A-p)^{2}}{(1-3 p)^{2}}\left|r-r_{0}\right|^{2-4 p}+\left[1-\frac{(A-p)^{2}}{(1-3 p)^{2}}\right]\left|r-r_{0}\right|^{2 p}\right. \\
+ & {\left.\left[A-p-\frac{(A-p)^{2}}{1-3 p}\right] \ln \left|r-r_{0}\right| \cdot\left|r-r_{0}\right|^{2 p}\right\} }
\end{aligned}
$$

which is not of the Kasner form if $(A-p)^{2} \neq(1-3 p)(A-p)$; the case $p=0$ is more fully discussed in sect. 4 . If two eigenvalues of $A$ are complex conjugate, with real part $\rho$ and imaginary part $\sigma$, the Lagrange interpolation formula

$$
f(A)=\sum_{i} f\left(p_{i}\right) \prod_{j \neq i} \frac{A-p_{j}}{p_{i}-p_{j}}
$$

\footnotetext{
${ }^{1}$ Note that, owing to the periodicity condition on $x^{1}=\varphi,(2.19)$ is not in general a globally defined coordinate transformation. As a consequence, the metric (2.18) is generically stationary, not static.
} 
leads to

$$
\begin{aligned}
\lambda= & M\left|r-r_{0}\right|^{2-4 \rho}+\left[N \cos \left(2 \sigma \ln \left|r-r_{0}\right|\right)\right. \\
& \left.+P \sin \left(2 \sigma \ln \left|r-r_{0}\right|\right)\right]\left|r-r_{0}\right|^{2 \rho}
\end{aligned}
$$

where the matrices $M, N, P$ are quadratic functions of $A$. The preceding cases are also discussed in [6].

In the second case $f=0$, eq. (2.5) is solved by

$$
\chi=A,
$$

where the constant matrix $A$ is now constrained by

$$
\operatorname{Tr} A=\operatorname{Tr} A^{2}=0,
$$

which implies the matrix relation

$$
A^{3}=(\operatorname{det} A) \mathbf{1}
$$

(conversely eq. (2.25) implies eq. (2.24), unless $A$ is proportional to the unit matrix 1). Equation (2.3) then leads to

$$
\lambda=C \mathrm{e}^{A r},
$$

where the matrix $C$ has the same signature and symmetry properties as before. Non-diagonal solutions of (2.25) may be classified [11 according to the rank $r(A)$ of the matrix $A$ :

(a) $\boldsymbol{r}(\boldsymbol{A})=3$. Then $A$ has the three eigenvalues $a, j a, j^{2} a$ (where $\left.j=\mathrm{e}^{2 i \pi / 3}\right)$, leading to

$$
\begin{aligned}
\lambda= & \frac{1}{3} C\left[\left(1+\bar{A}+\bar{A}^{2}\right) \mathrm{e}^{a r}+\left(2-\bar{A}-\bar{A}^{2}\right) \mathrm{e}^{-a r / 2} \cos \left(\frac{\sqrt{3}}{2} a r\right)\right. \\
& \left.+\sqrt{3}\left(\bar{A}-\bar{A}^{2}\right) \mathrm{e}^{-a r / 2} \sin \left(\frac{\sqrt{3}}{2} a r\right)\right],
\end{aligned}
$$

where $\bar{A}=a^{-1} A$; this space-time is of Petrov type $I$.

(b) $\boldsymbol{r}(\boldsymbol{A})=\mathbf{2}$, or $A^{3}=0, A^{2} \neq 0$. An example is

$$
A=\left(\begin{array}{rrr}
0 & 0 & 0 \\
0 & 0 & -1 \\
1 & 0 & 0
\end{array}\right), C=\left(\begin{array}{rrr}
0 & 1 & 0 \\
1 & 0 & 0 \\
0 & 0 & -1
\end{array}\right)
$$


leading to the metric

$$
\mathrm{d} s^{2}=-\frac{1}{2}(r \mathrm{~d} t+2 \mathrm{~d} z)^{2}+2 \mathrm{~d} t \mathrm{~d} \varphi+\mathrm{d} z^{2}-\mathrm{d} r^{2} .
$$

The Petrov type is $N$.

(c) $\boldsymbol{r}(\boldsymbol{A})=\mathbf{1}$, or $A^{2}=0$. Choosing

$$
A=\left(\begin{array}{lll}
0 & 0 & 0 \\
1 & 0 & 0 \\
0 & 0 & 0
\end{array}\right), C=\left(\begin{array}{rrr}
0 & 1 & 0 \\
1 & 0 & 0 \\
0 & 0 & -1
\end{array}\right),
$$

we obtain the metric 4

$$
\mathrm{d} s^{2}=r \mathrm{~d} t^{2}+2 \mathrm{~d} t \mathrm{~d} \varphi-\mathrm{d} z^{2}-\mathrm{d} r^{2} .
$$

In this case the curvature tensor vanishes, so that there must exist a local coordinate transformation which maps the metric into the Minkowski form

$$
\mathrm{d} s^{2}=\mathrm{d} U \mathrm{~d} V-\mathrm{d} Y^{2}-\mathrm{d} Z^{2}
$$

For the choice (2.30), this transformation is

$$
\begin{aligned}
& t=U, \quad \varphi=\frac{1}{2}\left[V+\frac{1}{6} U^{3}-U Y\right], \\
& r=Y-\frac{1}{4} U^{2}, \quad z=Z .
\end{aligned}
$$

However, because of the periodicity condition on the null coordinate $V$, the space-time of metric (2.31) is not globally equivalent to Minkowski spacetime.

(d) $\boldsymbol{r}(\boldsymbol{A})=\mathbf{0}$, i. e. $A=0$. The metric reduces to the "cylindrical Minkowski metric"

$$
\mathrm{d} s^{2}=\mathrm{d} t^{2}-R^{2} \mathrm{~d} \varphi^{2}-\mathrm{d} z^{2}-\mathrm{d} r^{2}
$$

this is again locally, but not globally, equivalent to Minkowski space-time. 


\section{The junction method for the hollow cylin- der problem}

We now return to our hollow cosmic string problem. Let us for convenience translate the radial coordinate $r$ (which is defined by the parametrization (2.1) only up to an additive constant) so that the surface $r=0$ corresponds to the cylinder surface. As in Frehland's analysis of the hollow cylinder problem [3], we use the junction method [5] to determine the space-time metric, i. e. the $3 \times 3$ matrix $\lambda(r)$, from the knowledge of the energy-momentum tensor $T^{\mu}{ }_{\nu}=\kappa^{-1} \mathcal{T}_{\nu}^{\mu} \delta(r)$ concentrated on the surface of the matter cylinder. The metric field $\lambda(r)$ may be written

$$
\lambda(r)=\lambda_{+}(r) \theta(r)+\lambda_{-}(r) \theta(-r),
$$

where the exterior and interior metrics $\lambda_{+}$and $\lambda_{-}$solving the vacuum Eistein equations may always be chosen so as to smoothly join along the cylinder $r=0$ :

$$
\lambda_{-}(0)=\lambda_{+}(0) .
$$

Because eq. (2.4) is satisfied everywhere, the energy-momentum component $T^{r}{ }_{r}$ vanishes on the cylinder from the first eq. (2.2). The remaining Einstein equations (2.2) then relate the discontinuity of the first derivative of the metric field to the surface energy- momentum $\mathcal{T}$ :

$$
\chi_{+}(0)-\chi_{-}(0)=2 \mathcal{T}-(\operatorname{Tr} \mathcal{T}) 1 .
$$

We choose the exterior metric to be the flat "spinning cosmic string" metric, generalizing the three-dimensional "spinning point particle" metric of [12],

$$
\mathrm{d} s_{+}^{2}=(\mathrm{d} t-\omega \mathrm{d} \varphi)^{2}-\alpha^{2}\left(r-r_{+}\right)^{2} \mathrm{~d} \varphi^{2}-\mathrm{d} z^{2}-\mathrm{d} r^{2},
$$

so that a distant observer will see the cylinder as a cosmic string of mass per unit length $M=2 \pi(1-\alpha) / \kappa$ and spin per unit length $J=2 \pi \omega / \kappa$. This metric is of the form (2.17), with $p_{1}=1, p_{0}=p_{2}=0$, and the basis vectors and one-forms

$$
\begin{aligned}
& n_{+}^{(0)}=\left(\begin{array}{l}
1 \\
0 \\
0
\end{array}\right), n_{+}^{(1)}=\left(\begin{array}{l}
\omega \\
1 \\
0
\end{array}\right), n_{+}^{(2)}=\left(\begin{array}{l}
0 \\
0 \\
1
\end{array}\right), \\
& \bar{n}_{+}^{(0)}=(1,-\omega, 0), \bar{n}_{+}^{(1)}=(0,1,0), \bar{n}_{+}^{(2)}=(0,0,1) .
\end{aligned}
$$


Of course the metric (3.4) is regular in the exterior region only if the conical singularity is virtual, $r_{+}<0$. As for the interior metric, we only assume that it has the generic form

$$
\lambda_{-}=C_{-} \exp \left[2 A_{-} \ln \left|r-r_{-}\right|\right],
$$

with the matrix $A_{-}$constrained by (2.11); the various 'exceptional' solutions (2.26) shall be recovered from (3.6) in the limit $r_{-} \rightarrow \infty$. Note that our assumptions are, in a certain sense, dual to those of Frehland [3], who assumed the interior metric to be Minkowskian, while the exterior metric was the general Kasner form (2.18).

Following Frehland, we assume the surface energy-momentum of the cylinder to be that of a uniformly rotating anisotropic perfect fluid:

$$
\mathcal{T}=\left(\begin{array}{cc}
\left(\mu-\tau_{\varphi}\right) u \otimes \bar{u}+\tau_{\varphi} \mathbf{1} & 0 \\
0 & \tau_{z}
\end{array}\right),
$$

with eigenvalues $\mu$ (surface energy density), $\tau_{\varphi}$ and $\tau_{z}$ (azimuthal and longitudinal stresses); here $\mathbf{1}$ is the $2 \otimes 2$ unit matrix. The local 2 -velocity $u$ is normalized by $\langle\bar{u}, u\rangle=1$, where $\bar{u}=u^{T} \lambda(0)$. We shall use the parametrization relative to the exterior basis

$$
u=\left(1-\beta^{2}\right)^{-1 / 2}\left(n_{+}^{(0)}+\Omega n_{+}^{(1)}\right), \bar{u}=\left(1-\beta^{2}\right)^{-1 / 2}\left(\bar{n}_{+}^{(0)}-\frac{\beta^{2}}{\Omega} \bar{n}_{+}^{(1)}\right),
$$

where $\Omega$ is the angular velocity of the rotating cylinder, and $\beta=-\alpha r_{+}|\Omega|$ is its linear velocity.

With these assumptions, the Einstein equations (3.3) read

$$
\begin{aligned}
& \frac{1}{r_{+}} n_{+}^{(1)} \otimes \bar{n}_{+}^{(1)}-\frac{1}{r_{-}} A_{-}= \\
& \quad\left(\begin{array}{cc}
\left(\tau_{\varphi}-\mu\right) u \otimes \bar{u}+\frac{\left(\mu-\tau_{\varphi}+\tau_{z}\right)}{2} 1 & 0 \\
0 & \frac{\left(\mu+\tau_{\varphi}-\tau_{z}\right)}{2}
\end{array}\right),
\end{aligned}
$$

leading to the form of the unknown matrix $A_{-}$in the basis $n_{+}^{(i)} \otimes \bar{n}_{+}^{(j)}$ :

$$
A_{-}=-r_{-}\left(\begin{array}{ccc}
\frac{\tau_{\varphi}-\mu}{1-\beta^{2}}+\frac{\left(\mu-\tau_{\varphi}+\tau_{z}\right)}{2} & \frac{\beta^{2}\left(\mu-\tau_{\varphi}\right)}{\Omega\left(1-\beta^{2}\right)} & 0 \\
\frac{\left(\tau_{\varphi}-\mu\right) \Omega}{1-\beta^{2}} & \frac{\beta^{2}\left(\mu-\tau_{\varphi}\right)}{1-\beta^{2}}+\frac{\left(\mu-\tau_{\varphi}+\tau_{z}\right)}{2}-\frac{1}{r_{+}} & 0 \\
0 & 0 & \frac{\left(\mu+\tau_{\varphi}-\tau_{z}\right)}{2}
\end{array}\right) .
$$


This matrix must obey the two constraints (2.11), which lead to the two relations

$$
\begin{aligned}
& \frac{1}{r_{+}}-\frac{1}{r_{-}}=\frac{1}{2}\left(\mu+\tau_{\varphi}+\tau_{z}\right), \\
& \beta^{2}=\frac{\tau_{\varphi}-r_{+}\left(\mu \tau_{\varphi}-\frac{1}{4}\left(\mu+\tau_{\varphi}-\tau_{z}\right)^{2}\right)}{\mu-r_{+}\left(\mu \tau_{\varphi}-\frac{1}{4}\left(\mu+\tau_{\varphi}-\tau_{z}\right)^{2}\right)} .
\end{aligned}
$$

These equations may be solved to give the metric parameters $r_{+}$and $r_{-}$in terms of the cylinder parameters $\mu, \tau_{\varphi}, \tau_{z}$ and $\beta$ :

$$
\begin{aligned}
& r_{+}=\frac{\tau_{\varphi}-\mu \beta^{2}}{\left(1-\beta^{2}\right)\left(\mu \tau_{\varphi}-\frac{1}{4}\left(\mu+\tau_{\varphi}-\tau_{z}\right)^{2}\right)}, \\
& r_{-}=\frac{4\left(\tau_{\varphi}-\mu \beta^{2}\right)}{\beta^{2}\left(3 \mu^{2}+\left(\tau_{\varphi}-\tau_{z}\right)^{2}\right)-\left(\left(\mu-\tau_{z}\right)^{2}+3 \tau_{\varphi}^{2}\right)} .
\end{aligned}
$$

Frehland's traceless condition [3] $\operatorname{Tr} \mathcal{T}=\mu+\tau_{\varphi}+\tau_{z}=0$ results from (3.11) if $r_{+}$and $r_{-}$happen to be equal. However, let us emphasize that this can only be a coincidence, as there is a priori no relation whatsoever between the locations of the virtual singularity $r_{+}$of the exterior metric and of the (real or virtual) apparent singularity $r_{-}$of the interior metric.

Solving the constraints (2.14) to determine the eigenvalues $p_{0}$ and $p_{1}$ of the matrix $A_{-}$from the eigenvalue $p_{2}$ (obvious from eq. (3.10)), and taking into account the first relation (3.11), we obtain

$$
\begin{aligned}
& p_{1}=\frac{1}{2} \frac{r_{-}}{r_{+}}\left(1-r_{+} \tau_{z} \mp \varepsilon \sqrt{\Delta}\right), \\
& p_{2}=-\frac{1}{2} r_{-}\left(\mu+\tau_{\varphi}-\tau_{z}\right),
\end{aligned}
$$

where

$$
\Delta=\left(1-r_{+} \tau_{z}\right)\left[1-2 r_{+}\left(\mu+\tau_{\varphi}\right)+r_{+} \tau_{z}\right],
$$

and $\varepsilon$ is a sign which shall be determined below. The corresponding arbitrarily normalized eigenvectors and associated one-forms are

$$
\begin{aligned}
& n_{-}^{(0)}=\gamma n_{+}^{(0)}+\frac{1}{2}(\eta \mp \varepsilon \sqrt{\Delta}) n_{+}^{(1)}, n_{-}^{(2)}=n_{+}^{(2)}, \\
& \bar{n}_{-}^{(0)}= \pm \delta^{-1}\left[\frac{1}{2}(\eta \pm \varepsilon \sqrt{\Delta}) \bar{n}_{+}^{(0)}-\gamma \bar{n}_{+}^{(1)}\right], \bar{n}_{-}^{(2)}=\bar{n}_{+}^{(2)},
\end{aligned}
$$


where we have put

$$
\gamma=\frac{r_{+}\left(\tau_{\varphi}-\mu\right)}{\Omega} \frac{\beta^{2}}{1-\beta^{2}}, \eta=1+r_{+}\left(\tau_{\varphi}-\mu\right) \frac{1+\beta^{2}}{1-\beta^{2}}, \delta=\frac{1-\beta^{2}}{1+\beta^{2}} \gamma \varepsilon \sqrt{\Delta} .
$$

To obtain the interior metric in the form (2.17), there remains to enforce the continuity conditions (3.2), which may be written as $\lambda_{-}(0) n_{-}^{(i)}=k_{i}\left|r_{-}\right|^{2 p_{i}} \bar{n}_{-}^{(i)}=$ $\lambda_{+}(0) n_{-}^{(i)}$, leading to

$$
\begin{aligned}
k_{0} & = \pm \frac{\varepsilon}{2} \alpha^{2} r_{+}^{2} \sqrt{\Delta}(\eta \mp \varepsilon \sqrt{\Delta})\left|r_{-}\right|^{-2 p_{0}}, \\
k_{2} & =-\left|r_{-}\right|^{-2 p_{2}}
\end{aligned}
$$

It can be checked that the product $k_{0} k_{1}$ is negative, as it should. The anholonomic coordinate $y_{0}$ in $(2.18)$ is timelike if $k_{0}$ is positive, which corresponds to the choice

$$
\varepsilon=\operatorname{sign} \eta
$$

The metric we have obtained is a solution to the hollow cylinder problem only if it is everywhere regular (except for the discontinuity (3.3) on the cylinder surface). Now the interior metric has an apparent singularity on the cylinder $r=r_{-}$. If $r_{-}<0$, a necessary condition for regularity is that the metric coefficients be analytic functions of $r$. This is possible if the exponents $p_{i}$ are integer, i. e. from eq. (2.14) if one of the $p_{i}$ is equal to 1 and the two others vanish. For $p_{1}=1$, the spatial sections have the Euclidean topology, while the two other cases $p_{0}=1$ and $p_{2}=1$ have non-Euclidean spatial topologies; we take the point of view that an observer outside the string should not rule out the possibility of exotic interior topologies. The other possibility is $r_{-}>0$, in which case the interior metric is regular for all values of the $p_{i}$. In this case (in which we also include the limit $r_{-} \rightarrow \infty$, leading to exceptional interior solutions), the global topology is that of a Lorentzian wormhole, with the two axes at infinity $r \rightarrow \pm \infty$. We study these various possibilities in the following two sections. 


\section{Solutions with an apparent singularity in- side the cylinder}

(a) $\boldsymbol{p}_{\mathbf{1}}=\mathbf{1}$. Then $p_{2}=0$ which, from the last equation (3.13), is possible only if the cylinder parameters are related by

$$
\tau_{z}=\mu+\tau_{\varphi}
$$

In this case (as well as in case (b) $p_{0}=1$, which also implies $p_{2}=0$ ), the matrix $A_{-}$of eq. (3.10) is really two-dimensional[ so that, taking into account the continuity of the metric tensor, the interior metric reduces to

$$
\mathrm{d} s_{-}^{2}=\lambda_{-(2) a b}(r) \mathrm{d} x^{a} \mathrm{~d} x^{b}-\mathrm{d} z^{2}-\mathrm{d} r^{2} .
$$

The global space-time is then the product of the $z$ axis by the three-dimensional space-time generated by a rotating closed string of energy density $\mu$ and stress $\tau_{\varphi}$ [四].

The first equation (3.13) with $p_{0}=0, p_{1}=1$ leads to the conditions

$$
1-r_{+} \tau_{z}=\varepsilon \sqrt{\Delta}=\frac{r_{+}}{r_{-}} \text {. }
$$

As $r_{+}$and $r_{-}$are both negative, it follows that $\varepsilon=+1$. The interior metric may be written in the form (2.18),

$$
\mathrm{d} s_{-}^{2}=\mathrm{d} t^{\prime 2}-\left(r-r_{-}\right)^{2} \mathrm{~d} \varphi^{\prime}-\mathrm{d} z^{2}-\mathrm{d} r^{2},
$$

with

$$
\begin{aligned}
& \mathrm{d} t^{\prime}=\sqrt{k_{0}} \delta^{-1}\left[\frac{1}{2}(\sqrt{\Delta}+\eta) \mathrm{d} t-\left(\frac{1}{2}(\sqrt{\Delta}+\eta) \omega+\frac{\delta}{\sqrt{\Delta}}\right) \mathrm{d} \varphi\right] \\
& \mathrm{d} \varphi^{\prime}=\sqrt{-k_{1}} \delta^{-1}\left[\frac{1}{2}(\sqrt{\Delta}-\eta) \mathrm{d} t-\left(\frac{1}{2}(\sqrt{\Delta}-\eta) \omega-\frac{\delta}{\sqrt{\Delta}}\right) \mathrm{d} \varphi\right] .
\end{aligned}
$$

However this coordinate transformation is consistent with the periodicity condition on $\varphi$ only if the time $t^{\prime}$ does not depend on $\varphi$ and if the angle $\varphi^{\prime}$ has the same period $2 \pi$ as $\varphi$, i. e.

$$
\frac{1}{2}(\eta+\sqrt{\Delta}) \omega+\frac{\delta}{\sqrt{\Delta}}=0, \quad \sqrt{-k_{1}} \delta^{-1}\left[\frac{1}{2}(\eta-\sqrt{\Delta}) \omega+\frac{\delta}{\sqrt{\Delta}}\right]=1
$$

\footnotetext{
${ }^{2}$ It then follows from eq. (2.11) that $A_{-}^{2}=A_{-}$, so that the interior metric can be put in the Kasner form even though the eigenvalue 0 is degenerate.
} 
(if these conditions are not satisfied, then the interior metric is a spinning cosmic string metric, with a conical singularity at $r=r_{-}$). The transformation (4.5) then reduces to a time dilation coupled with a uniform frame rotation of angular velocity $-\omega / r_{-}^{2}$.

Because the interior metric (4.4) is Minkowskian, the proper radius of the cylinder $r=0$ is unambiguously identified as $\left|r_{-}\right|$, which is different from the two possible exterior definitions $\left|r_{+}\right|$and $\alpha\left|r_{+}\right|$. Taking the independent parameters of the cylinder to be $\left|r_{-}\right|, \mu$ and $\tau_{\varphi}, \tau_{z}$ is given by (4.1), while from eqs. (3.11) the linear rotation velocity of the cylinder is

$$
\beta=\frac{\tau_{\varphi}\left(1+r_{-} \tau_{\varphi}\right)}{\mu\left(1+r_{-} \mu\right)}
$$

(positive from eq. (4.9) below). If such a fine tuning between the cylinder parameters is not achieved, then the centrifugal energy of the rotating cylinder does not balance its azimuthal stress, and the cylinder either contracts or expands. The external metric parameter $r_{+}$is, from the first eq. (3.11),

$$
r_{+}=\frac{r_{-}}{1+r_{-}\left(\mu+\tau_{\varphi}\right)} .
$$

The remaining exterior metric parameters $\alpha$ and $\omega$ are then obtained from the regularity conditions (4.6),

$$
\begin{aligned}
\alpha^{2} & =\left(1+r_{-} \mu\right)\left(1+r_{-} \tau_{\varphi}\right)\left(1+r_{-}\left(\mu+\tau_{\varphi}\right)\right), \\
\omega^{2} & =\frac{r_{-}^{4} \mu \tau_{\varphi}}{1+r_{-}\left(\mu+\tau_{\varphi}\right)}
\end{aligned}
$$

(to obtain this last relation, we have also used the definition $\beta=-\alpha r_{+}|\Omega|$ ). Combining eqs. (4.8) and (4.9), we obtain the relation

$$
\alpha^{2} r_{+}^{2}-\omega^{2}=r_{-}^{2} \text {. }
$$

In (4.10) we recognize the continuity condition (3.2) for the metric component $g_{\varphi \varphi}$ (which is unaffected by a uniform frame rotation). A consequence is that $g_{\varphi \varphi}$ stays negative outside as well as inside the cylinder $r=0$ so that, contrary to the case of the spinning cosmic string space-time [12], the global space-time generated by the rotating cylinder does not contain closed timelike curves. This is a very satisfactory feature of this solution. 
We still have to enforce the inequalities $r_{+}<0, r_{-}<0, \omega^{2} \geq 0$ (from which follows $\alpha^{2}>0$ ) and $\beta<1$. These inequalities lead to two possible domains of parameter values. The first possibility

$$
0 \leq \tau_{\varphi}<\mu, \quad \mu+\tau_{\varphi}<\frac{1}{\left|r_{-}\right|}
$$

also implies

$$
0 \leq \tau_{\varphi}<\beta^{2} \mu,
$$

and leads to the total cylinder mass per unit length

$$
M=\frac{2 \pi}{\kappa}(1-\alpha)>2 \pi\left|r_{-}\right| \frac{\mu}{\kappa} .
$$

The second possibility $\mu<\tau_{\varphi} \leq 0$, which also implies $\beta \mu<\tau_{\varphi}<\beta^{2} \mu$, must be excluded as it leads to a negative net mass per unit length $M<$ $2 \pi\left|r_{-}\right| \mu / \kappa<0$.

(b) $\boldsymbol{p}_{\mathbf{0}}=\mathbf{1}$. In this case again, $p_{2}=0$ implies the relation (4.1), while eq. (4.3) is here replaced by

$$
1-r_{+} \tau_{z}=-\varepsilon \sqrt{\Delta}=\frac{r_{+}}{r_{-}}
$$

from which follows $\varepsilon=-1$. This last condition is equivalent to the inequality $k_{0-}>0$, which may be written

$$
\left(1-r_{+} \mu\right)\left(1-r_{+} \tau_{\varphi}\right)<0
$$

Taking into account eqs. (4.7) and (4.8), which hold again in this case, the inequality (4.15) results in the condition

$$
0 \leq-\beta \mu<\tau_{\varphi}
$$

The conditions (4.9) have no equivalent here. The reason is that, in this case, a suitable uniform frame rotation on the interior metric determined in the previous section leads to the metric [4]

$$
\mathrm{d} s_{-}^{2}=\nu^{2}\left(r-r_{-}\right)^{2}\left(\mathrm{~d} t-\omega_{-} \mathrm{d} \varphi^{\prime}\right)^{2}-R^{2} \mathrm{~d} \varphi^{\prime 2}-\mathrm{d} z^{2}-\mathrm{d} r^{2} .
$$


The apparent singularity of this metric at $r=r_{-}$is actually a cylindrical horizon. The corresponding space-time is flat, as may be shown by carrying out the local Rindler-like [13] transformation

$$
\begin{aligned}
\bar{t} & =\left(r-r_{-}\right) \sinh \left[\nu\left(t-\omega_{-} \varphi^{\prime}\right)\right], \\
\bar{r} & =\left(r-r_{-}\right) \cosh \left[\nu\left(t-\omega_{-} \varphi^{\prime}\right)\right], \\
\bar{\varphi} & =\varphi^{\prime}, \quad \bar{z}=z,
\end{aligned}
$$

which maps the domain $r_{-} \leq r \leq 0$ of the interior space-time on one of the two domains $I\left(0 \leq \bar{r}^{2}-\bar{t}^{2} \leq r_{-}^{2}\right)$ of the Minkowski cylinder

$$
\mathrm{d} s_{-}^{2}=\mathrm{d} \bar{t}^{2}-\mathrm{d} \bar{r}^{2}-R^{2} \mathrm{~d} \bar{\varphi}^{2}-\mathrm{d} \bar{z}^{2} .
$$

The maximal extension of the metric (4.17) is of course diffeomorphic to the entire Minkowski cylinder. However, the maximally extended interior spacetime is not globally equivalent to the Minkowski cylinder, for two reasons. The first is that the Rindler time $t-\omega_{-} \varphi^{\prime}$ in (4.17) has a helical structure [12]; a consequence is the occurence of closed timelike curves outside the cylinder $\nu^{2} \omega_{-}^{2}\left(r-r_{-}\right)^{2}=R^{2}$. The second reason is the inequality $r \leq 0$, which results in the truncation $\bar{r} \leq\left(r_{-}^{2}+\bar{t}^{2}\right)^{1 / 2}$ of the Minkowski cylinder.

The metric (4.17) is invariant under the discrete symmetry $r-r_{-} \rightarrow r_{-}-r$. The full space-time geometry is invariant under this symmetry if the matter cylinder at $r=0$ is echoed by a mirror image cylinder at $r=2 r_{-}$. The Penrose diagram of this space-time is shown in Fig. 1. The two mirrorsymmetric cylinders separate space-time into three regions, two symmetrical exterior regions with a truncated spinning cosmic string geometry, and an interior region with a truncated spinning Rindler cylinder geometry (the truncated maximal extension of the metric (4.17)). An observer falling inside the stationary cylinder may send lightlike signals to the outside until he crosses the horizon $r=r_{-}$. Thereafter the observer continues falling through flat space-time towards interior timelike infinity, while the cylindrical walls now seem, in the Minkowskian frame (4.19), to expand according to $\bar{r}=$ $\pm\left(r_{-}^{2}+\bar{t}^{2}\right)^{1 / 2}$. The attempts of the trapped observer to communicate with the outside are now doomed, as his signals $\bar{r}-\bar{t}=$ constant never catch up with the receding walls, and end up at interior lightlike infinity $\mathcal{I}_{i n}^{+}$. The future Rindler region II $\left(\bar{r}^{2}-\bar{t}^{2}<0\right)$ is thus, for an external observer, a cylindrical black hole with event horizon $r=r_{-}$[14]. 
We should emphasize here that this black hole structure arises if the parameters of the matter cylinder are constrained by (4.1) and (4.16), so that the matter making up the cylinder has negative energy density $\mu$ (even in the static limit $\beta \rightarrow 0$ ) and positive stresses $\tau_{\varphi}$ and $\tau_{z}$. The exotic character of this matter shows up in the dynamics (the fate of a falling observer), but not in the exterior space-time geometry, which is the spinning cosmic string geometry (3.4) with parameters $\alpha$ and $\omega$ which may be arbitrarily chosen (only $r_{+}$is determined from the string parameters by eq. (3.12)). We may thus choose these parameters so as to ensure that the total (matter + gravitational) mass per unit length $M=2 \pi(1-\alpha) / \kappa$ is positive $(\alpha<1)$ and that there are no timelike curves $\left(|\omega|<\alpha\left|r_{+}\right|\right.$, which also ensures the absence of closed timelike curves inside the matter cylinder, because of the continuity of $\left.g_{\varphi \varphi}\right)$.

(c) $\boldsymbol{p}_{\mathbf{2}}=\mathbf{1}$. From eqs. (3.13) and (3.11), this occurs if the metric parameters $r_{+}$and $r_{-}$are related to the longitudinal tension $\tau_{z}$ by

$$
r_{+}=\frac{1}{\tau_{z}}, r_{-}=-\frac{2}{\mu+\tau_{\varphi}-\tau_{z}}
$$

while the linear rotation velocity $\beta$ of the string is

$$
\beta=\left|\frac{\mu-\tau_{\varphi}-\tau_{z}}{\mu-\tau_{\varphi}+\tau_{z}}\right| .
$$

The inequalities $r_{+}<0, r_{-}<0\left(r_{-}\right.$finite) and $\beta<1$ are equivalent to the conditions

$$
\mu<\tau_{\varphi}, \quad \tau_{z}<0, \quad \tau_{z}<\mu+\tau_{\varphi}
$$

on the cylinder parameters.

On account of eqs. (4.20) and (4.21), the matrix $A_{-}$of eq. (3.10) reduces to

$$
A_{-}=\left(\begin{array}{ccc}
a & \varepsilon^{\prime} \frac{\alpha}{\tau_{z}} a & 0 \\
-\varepsilon^{\prime} \frac{\tau_{z}}{\alpha} a & -a & 0 \\
0 & 0 & 1
\end{array}\right)
$$

where

$$
a=\frac{\tau_{z}^{2}-\left(\mu-\tau_{\varphi}\right)^{2}}{2 \tau_{z}\left(\mu+\tau_{\varphi}-\tau_{z}\right)}
$$


and $\varepsilon^{\prime}=\operatorname{sign}\left(\Omega\left[\tau_{z}^{2}-\left(\mu-\tau_{\varphi}\right)^{2}\right]\right)$. According to eq. (2.20), the resulting matrix $\lambda_{-}$is

$$
\lambda_{-}=C_{-}\left[1-A_{-}^{2}+\left(A_{-}-A_{-}^{2}\right) \ln \left(r-r_{-}\right)^{2}+A_{-}^{2}\left(r-r_{-}\right)^{2}\right],
$$

with

$$
A_{-}^{2}=\left(\begin{array}{lll}
0 & 0 & 0 \\
0 & 0 & 0 \\
0 & 0 & 1
\end{array}\right) .
$$

If $a \neq 0$, the matrix $A_{-}-A_{-}^{2}$ is non zero so that the metric corresponding to (4.25) is non-Kasner. Using the continuity condition (3.2) to determine the matric $C_{-}$, we obtain the interior metric

$$
\begin{aligned}
\mathrm{d} s_{-}^{2}= & (\psi+1) \mathrm{d} t^{2}-2\left(\omega^{\prime} \psi+\omega\right) \mathrm{d} \varphi \mathrm{d} t+\left[\omega^{\prime 2} \psi+\omega^{\prime}\left(2 \omega-\omega^{\prime}\right)\right] \mathrm{d} \varphi^{2} \\
& -\frac{\left(r-r_{-}\right)^{2}}{r_{-}^{2}} \mathrm{~d} z^{2}-\mathrm{d} r^{2}
\end{aligned}
$$

with

$$
\psi=a \ln \frac{\left(r-r_{-}\right)^{2}}{r_{-}^{2}}, \omega^{\prime}=\omega+\varepsilon^{\prime} \alpha r_{+},
$$

The only non-zero components of the curvature tensor following from (4.27) are

$$
R_{\beta 2}^{\alpha 2}=-R_{\beta 3}^{\alpha 3}=-\frac{1}{\left(r-r_{-}\right)^{2}} A_{\beta}^{\alpha}
$$

$(\alpha, \beta=0,1)$, of Petrov type $I$. Obviously the curvature invariants vanish, so that the singularity of the interior metric at $r=r_{-}$is a non-scalar curvature singularity [15]. Because of this singularity, the metric (4.27) is not a regular solution to the hollow cylinder problem. However, as we shall now show by studying the geodesic motion of a test particle in this metric, for a certain range of the cylinder parameters the singularity $r=r_{-}$is 'harmless', i. e. it is avoided by all timelike and lightlike geodesics. The geodesic equation in a stationary cylindrically symmetric metric (2.1) may be integrated to

$$
\left(\frac{\mathrm{d} r}{\mathrm{~d} \tau}\right)^{2}-\Pi^{T} \lambda^{-1} \Pi+\eta=0,
$$

where $\eta=+1,0$ or $-1, \tau$ is an affine parameter, and the generalized momenta

$$
\Pi_{a}=\lambda_{a b} \frac{\mathrm{d} x^{a}}{\mathrm{~d} \tau}
$$


are constants of motion. In the case of the metric (4.27), equation (4.30) reads

$$
\left(\frac{\mathrm{d} r}{\mathrm{~d} \tau}\right)^{2}+p^{2}(\psi+1)+2 p \Pi_{0}+\Pi_{2}^{2} \frac{r_{-}}{\left(r-r_{-}\right)^{2}}+\eta=0,
$$

with $p=-\left(\omega^{\prime} \Pi_{0}+\Pi_{1}\right) \varepsilon^{\prime} / \alpha r_{+}$. All the geodesics are deflected away from $r=$ $r_{-}$by the potential barrier in $\left(r-r_{-}\right)^{-2}$, unless $\Pi_{2}=0$. If $\Pi_{2}=0$, then for $a>0$ almost all the geodesics terminate at the singularity $r=r_{-}(\psi \rightarrow-\infty)$. However for $a<0$ all the geodesics are deflected away by the logarithmic potential barrier $p^{2} \psi$, unless $p=0$. In this last case ( which excludes timelike geodesics $\eta=1)$, only spacelike geodesics $(\eta=-1)$ extend to $r=r_{-}$. We conclude that test particles are always repelled by the singularity $r=r_{-}$if $a<0$, i. e.

$$
\mu>\tau_{\varphi}+\tau_{z}
$$

(note that the constraints (4.22) and (4.33) allow the possibility of a positive energy density $\mu$ ). An unpleasant corollary of the choice $a<0$ is that for $\psi$ large enough the interior geometry (4.27) admits closed timelike lines. This pathology does not occur if the parameters of the exterior metric are constrained by $\omega^{\prime}=0\left(\omega=-\varepsilon^{\prime} \alpha r_{+}\right)$, which also excludes closed timelike lines outside the cylinder. However the interior geometry still admits for $\omega^{\prime}=0$ closed lightlike lines, the closed null geodesics $\mathrm{d} t=\mathrm{d} z=\mathrm{d} r=0$ $\left(\Pi_{1}=\Pi_{2}=0\right)$.

If now $a=0$, which from eq. (4.21) implies

$$
\tau_{z}=\mu-\tau_{\varphi}, \beta=0
$$

the matrix $A_{-}$assumes the diagonal form (4.26), and the interior metric is simply

$$
\mathrm{d} s_{-}^{2}=(\mathrm{d} t-\omega \mathrm{d} \varphi)^{2}-\alpha^{2} r_{+}^{2} \mathrm{~d} \varphi^{2}-\frac{\left(r-r_{-}\right)^{2}}{r_{-}^{2}} \mathrm{~d} z^{2}-\mathrm{d} r^{2} .
$$

This metric has a conical singularity at $r=r_{-}$, unless the variable $z /\left|r_{-}\right|$is an angle, i. e. unless $z$ and $z+2 \pi\left|r_{-}\right|$are identified. To be consistent, such an identification should be carried over to the exterior metric, so that the 'longitudinal' dimension $z$ in (3.4) is actually wrapped up around a circle. This implies that our hollow cosmic string is not cylindrical but toroidal, 
with small radius $\alpha\left|r_{+}\right|=-\alpha / \tau_{z}$ and large radius $\left|r_{-}\right|=1 / \tau_{\varphi}$. Of course, space-time around such a torus can only be approximately described by the cylindrical metric (3.4), a necessary condition for such an approximation being $\left|r_{-}\right| \gg \alpha\left|r_{+}\right|$, i. e.

$$
\alpha \tau_{\varphi} \ll-\tau_{z} .
$$

The hollow cosmic string then connects two three-dimensional spaces where the torus $r=0$ is embedded in two different ways, the exterior space with approximate metric (3.4), and the interior space (4.35) where the torus now has $\left|r_{-}\right|$as 'small' radius and $\alpha\left|r_{+}\right|$as 'large' radius.

Weak-field self-gravitating circular cosmic strings have been constructed in [16]. Our various hollow cosmic string solutions can presumably be approximately extended to the case of circular cosmic strings by compactifying the longitudinal dimension $z$. The solution $p_{2}=1, a=0$ is unique in that it spontaneously compactifies. It is weak-field if $\alpha \simeq 1$, in which case $0<\tau_{\varphi} \ll-\mu$ from eqs. (4.34) and (4.36), so that the energy density on the torus is negative. Contrary to the result of [16], the total mass of this hollow cosmic string

$$
2 \pi\left|r_{-}\right| M=(1-\alpha) \frac{4 \pi^{2}}{\kappa}\left|r_{-}\right|
$$

is different from the matter energy $4 \pi^{2}\left|r_{-}\right| \alpha\left|r_{+}\right| \mu / \kappa \simeq-\alpha\left(4 \pi^{2} / \kappa\right)\left|r_{-}\right|$. The corresponding gravitational radius $(1-\alpha)(\pi / 2)\left|r_{-}\right|$is, in the weak-field case $\alpha \simeq 1$, negligible (as it should) with respect to the radius $\left|r_{-}\right|$of the matter distribution.

\section{Solutions without an apparent singularity}

The interior metric (3.6) is regular for $r \leq 0$, whatever the values of the Kasner exponents $p_{i}$, if $r_{-}>0$. In this case the two regularity conditions $r_{+}<0, r_{-}>0$ constrain the possible domains of values of the cylinder parameters. To simplify the notation, let us define

$$
x=\mu+\tau_{\varphi}, \quad y=\mu-\tau_{\varphi}, \quad z=\tau_{z} .
$$

We have, from eqs. (3.11) and (3.12),

$$
r_{+}=\frac{2}{t}, \quad r_{-}=\frac{2}{t-x-z},
$$


with

$$
t=\frac{y^{2}+z^{2}-2 x z}{h^{-1} y-x}, h=\frac{1-\beta^{2}}{1+\beta^{2}} .
$$

Then the conditions $r_{+}<0, r_{-}>0$ read

$$
x+z \leq t \leq 0 .
$$

The nature of the interior geometry depends on the value of

$$
p_{2}=\frac{z-x}{t-x-z} .
$$

For $-1 / 3 \leq p_{2} \leq 0$ the other two exponents $p_{0}$ and $p_{1}$ are both positive; for $0 \leq p_{2} \leq 1$, one of the other exponents is positive and the other is negative; finally, for $p_{2}<-1 / 3$ or $p_{2}>1$, the exponents $p_{0}$ and $p_{1}$ are complex conjugate. A lengthy analysis [17] of these inequalities together with the regularity conditions (5.4) leads to the domains of parameter values shown in Table 1, where we have put

$$
\begin{aligned}
& k=\frac{1-\beta}{1+\beta}, \quad l=\frac{\sqrt{3}\left(1-\beta^{2}\right)+2 \beta}{\sqrt{3}\left(1-\beta^{2}\right)-2 \beta} \\
& y_{0}=\sqrt{z(2 x-z)}, \quad \bar{y}_{ \pm}=k^{ \pm 1}(2 x-z), \quad \tilde{y}_{ \pm}=k^{ \pm 1} z \\
& y_{ \pm}=\frac{1}{2}\left[h^{-1}(x+z) \pm \sqrt{\left(h^{-2}-1\right)(x+z)^{2}-3(x-z)^{2}}\right] .
\end{aligned}
$$

The various domains $\mathrm{c}$ are related to the corresponding domains $\mathrm{b}$ by the involution $(x, y, z) \rightarrow(x, y, 2 x-z)$.

Because the interior metric is regular for all values of $r \leq 0$, the spacetimes of this section have the wormhole topology, with two axes at infinity $r \rightarrow \pm \infty$. To ascertain whether these wormholes are traversable or not, we study the motion of a test particle in the interior metric, given by the integrated geodesic equation (4.30) with $\eta=+1$ or 0 .

When the Kasner exponents $p_{i}$ are all real (domains b and c), this equation reads

$$
\left(\frac{\mathrm{d} r}{\mathrm{~d} \tau}\right)^{2}-\sum_{i} k_{i}^{-1}\left\langle\Pi, n_{-}^{(i)}\right\rangle^{2}\left|r-r_{-}\right|^{-2 p_{i}}+\eta=0 .
$$

For large negative $r$, the leading term in the effective potential comes from the Kasner term with negative exponent $p_{i}$ (for $-1 / 3 \leq p_{2} \leq 1$, one and only 
a) $p_{2} \leq-1 / 3$

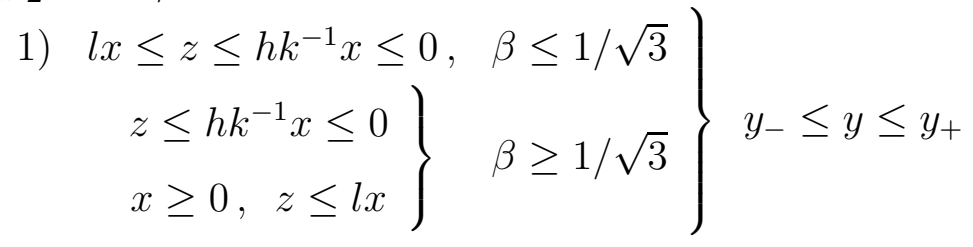

2) $h k^{-1} x \leq z \leq x \leq 0, \quad y_{-} \leq y \leq \bar{y}_{-}$or $y_{+} \leq y \leq \bar{y}_{+}$

3) $2 x \leq z \leq h k^{-1} x \leq 0, \quad \bar{y}_{-} \leq y \leq \bar{y}_{+}$

b) $-1 / 3 \leq p_{2} \leq 0$

1) $2 x \leq z \leq h k^{-1} x \leq 0, \quad-y_{0} \leq y \leq \bar{y}_{-}$

2) $h k^{-1} x \leq z \leq x \leq 0, \quad \bar{y}_{-} \leq y \leq-y_{0}$

3) $2 x \leq z \leq x \leq 0, \quad \bar{y}_{+} \leq y \leq y_{0}$

c) $\mathbf{0} \leq \boldsymbol{p}_{\mathbf{2}} \leq \mathbf{1}$

1) $h k x \leq z \leq 0, \quad-y_{0} \leq y \leq \tilde{y}_{-}$

2) $x \leq z \leq h k x \leq 0, \quad \tilde{y}_{-} \leq y \leq-y_{0}$

3) $x \leq z \leq 0, \quad \tilde{y}_{+} \leq y \leq y_{0}$

d) $\mathbf{1} \leq \boldsymbol{p}_{\mathbf{2}}$
1) $x \leq z \leq h k x \leq 0$, $y_{-} \leq y \leq \tilde{y}_{-}$
2) $x \leq 0, h k x \leq z \leq l^{-1} x \begin{cases}\beta \leq 2-\sqrt{3}, & \tilde{y}_{-} \leq y \leq y_{-} \\ \beta \geq 2-\sqrt{3}, & y_{-} \leq y \leq y_{+}\end{cases}$
3) $x \leq z \leq l^{-1} x \leq 0$, $x \leq z \leq h k x \leq 0$, $\left.\begin{array}{l}\beta \leq 2-\sqrt{3} \\ \beta \geq 2-\sqrt{3}\end{array}\right\} y_{+} \leq y \leq \tilde{y}_{+}$
4) $l^{-1} x \leq z \leq 0$ $h k x \leq z \leq 0$, $\left.\begin{array}{l}\beta \leq 2-\sqrt{3} \\ \beta \geq 2-\sqrt{3}\end{array}\right\} \tilde{y}_{-} \leq y \leq \tilde{y}_{+}$

Table 1: The various domains of the cylinder parameter values leading to regular wormhole solutions 
one of the $p_{i}$ is negative). The wormhole is traversable if this term is timelike $\left(p_{0}<0\right)$, and is generically not traversable if this term is spacelike $\left(p_{1}<0\right.$ or $p_{2}<0$, so that $\left.p_{0}>0\right)$. The sign of $p_{0}$ depends both on the sign of $p_{2}$ and on the sign $\varepsilon$ in (3.13), which we can show [17] to be negative in the cases $\mathrm{b} 1, \mathrm{~b} 2, \mathrm{c} 1, \mathrm{c} 2$, and positive in the cases b3, c3. We conclude that in the cases $\mathrm{b}$ and $\mathrm{c} 3, p_{0}>0$ and the test particle is generically reflected by a potential barrier in $\left|r-r_{-}\right|^{-2 p_{j}}(j=1$ or 2$)$, except if the constant of the motion $\left\langle\Pi, n_{-}^{(j)}\right\rangle$ happens to vanish; in this last case massive particles $(\eta=+1)$ are again reflected to exterior spacelike infinity, while massless particles $(\eta=0)$ can travel to interior spacelike infinity $r \rightarrow-\infty$ in the cases b1, b2 and in the case $\mathrm{c} 3$ if $p_{2}>2 / 3$. In the cases $\mathrm{c} 1$ and $\mathrm{c} 2, p_{0}<0$ and the particle falls into the potential well towards interior spacelike infinity. A special case of these traversable wormholes is the symmetrical wormhole [4] $p_{2}=0$ with $x=z, y=h^{-1} x$ (belonging to the case $\mathrm{c} 2$ ). Then the interior metric has the same form

$$
\mathrm{d} s_{-}^{2}=\left(\mathrm{d} t-\omega_{-} \mathrm{d} \varphi^{\prime}\right)^{2}-\alpha_{-}^{2}\left(r-r_{-}\right)^{2} \mathrm{~d} \varphi^{\prime 2}-\mathrm{d} r^{2}
$$

as the exterior metric (3.4), with $\alpha_{-}=\alpha_{+}$arbitrary, $\omega_{-}=-\omega_{+}, r_{-}=-r_{+}$, so that the full space-time admits the discrete isometry $t \rightarrow t, \varphi \rightarrow-\varphi^{\prime}$, $r \rightarrow-r$, which exchanges the two axes at spacelike infinity.

The Kasner exponents $p_{0}$ and $p_{1}$ are complex conjugate in the domains a and $\mathrm{d}$. The matrix $\lambda^{-1}$ is obtained by inverting eq. (2.22), with $\rho=\left(1-p_{2}\right) / 2$, $\sigma^{2}=\left(p_{2}-1\right)\left(3 p_{2}+1\right) / 4$, leading to the geodesic equation

$$
\left(\frac{\mathrm{d} r}{\mathrm{~d} \tau}\right)^{2}+b\left|r-r_{-}\right|^{-2 p_{2}}+c \sin \left(2 \sigma \ln \left|r-r_{-}\right|+d\right)\left|r-r_{-}\right|^{p_{2}-1}+\eta=0,
$$

with $b \geq 0$. Incoming test particles are always reflected back to exterior infinity by the effective potential in (5.9), generically by the potential barrier $b\left|r-r_{-}\right|^{-2 p_{2}}$ for $p_{2}<-1 / 3$, or by the oscillating term in $\left|r-r_{-}\right|^{p_{2}-1}$ for $p_{2}>1$.

Up to now, we have assumed that the interior metric has the generic form (3.6), excluding the exceptional metrics (2.26). For an exceptional metric, the matrix $\left(1 / r_{-}\right) A_{-}$in $(3.10)$ should be replaced by $(1 / 2) A_{-}$, where $A_{-}$has the form

$$
A_{-}=a\left(\begin{array}{cc}
A_{2} & 0 \\
0 & 1
\end{array}\right)
$$


with

$$
\operatorname{Tr} A_{2}=\operatorname{Tr} A_{2}^{2}=-1
$$

from eq. (2.24), and

$$
a=\tau_{z}-\mu-\tau_{\varphi} .
$$

The constraints (5.11) lead to the two relations

$$
\begin{aligned}
& \frac{1}{r_{+}}=\frac{1}{2}\left(\mu+\tau_{\varphi}+\tau_{z}\right), \\
& \beta^{2}=\frac{\left(\mu-\tau_{z}\right)^{2}+3 \tau_{\varphi}^{2}}{3 \mu^{2}+\left(\tau_{\varphi}-\tau_{z}\right)^{2}},
\end{aligned}
$$

which may also be obtained from eq. (3.11) by taking the limit $r_{-} \rightarrow \infty$; the conditions $r_{+}<0, \beta<1$ are satisfied if

$$
\mu<\tau_{\varphi}<-\mu-\tau_{z}
$$

If $a \neq 0\left(\tau_{z} \neq \mu+\tau_{\varphi}\right)$ we obtain, using (2.27) and (5.10), the interior metric

$$
\mathrm{d} s_{-}^{2}=\mathrm{e}^{-a r / 2}\left(C_{2} \mathrm{e}^{B(\sqrt{3} / 2) a r}\right)_{a b} \mathrm{~d} x^{a} \mathrm{~d} x^{b}-\mathrm{e}^{a r} \mathrm{~d} z^{2}-\mathrm{d} r^{2},
$$

where $C_{2}$ and $B$ are $2 \times 2$ matrices, with $B^{2}=-1$. Again, we can show that incoming test particles are reflected back to exterior infinity by an exponentially rising potential barrier if $a>0$, an oscillating potential barrier if $a<0$. In the special case $a=0$, corresponding to

$$
\tau_{\varphi}= \pm \beta \mu, \tau_{z}=(1 \pm \beta) \mu,
$$

with $\mu<0, A$ is of rank 1 and the interior metric [4]

$$
\begin{aligned}
\mathrm{d} s_{-}^{2}= & (\gamma r+1)(\mathrm{d} t-\omega \mathrm{d} \varphi)^{2}-2 \varepsilon^{\prime \prime} \alpha r_{+} \gamma r(\mathrm{~d} t-\omega \mathrm{d} \varphi) \mathrm{d} \varphi \\
& +\alpha^{2} r_{+}^{2}(\gamma r-1) \mathrm{d} \varphi^{2}-\mathrm{d} z^{2}-\mathrm{d} r^{2},
\end{aligned}
$$

(with $\gamma= \pm 2 \beta \mu /(1 \pm \beta), \varepsilon^{\prime \prime}= \pm \operatorname{sign} \Omega$ ) is flat. In the case of the sign + in (5.16), $\gamma<0$ and incoming test particles are reflected by a linearly rising potential, while for the sign - the particles fall towards interior infinity with constant proper acceleration [4]. Finally, if $\beta=0$ in (5.16), $A$ is of rank 0 and the interior metric reduces to the Minkowski cylinder (2.34). 


\section{Discussion}

We have determined and analyzed the various possible regular space-time metrics inside an infinite rotating hollow cylinder, the metric outside the cylinder being chosen of the spinning cosmic string type. While the analogous spherically symmetric problem admits a unique solution, a traversable wormhole made of two Schwarzschild space-times with a spherical matter shell as common boundary [18], the variety of solutions we have obtained here is a striking illustration of the non-existence of a Birkhoff theorem in the case of cylindrical symmetry. These solutions include a space-time with topologically Euclidean spatial sections, eq. (4.4), a black hole solution, eq. (4.17), a quasi-regular solution, eq. (4.27) with $a<0$, as well as the wormhole solutions of section 5. The special solution (4.35) might approximately describe spontaneous compactification of the cylinder to a torus.

Although the exterior metric is the same for all these solutions, an observer at exterior spatial infinity can gain some information on the values of the cylinder parameters by aiming beams of test particles towards the cylinder. In the case of the black-hole solution, as well as for the traversable wormhole solutions $\mathrm{c} 1$ and $\mathrm{c} 2$ of section 5 and the exceptional solution (5.17) with the sign -, all the incoming test particles disappear inside the cylinder. Some photons may be absorbed by the cylinder in the case of the wormhole solutions b and c3, while all incoming test particles are reflected back to the outside in the other cases. If we insist on the positivity of the cylinder energy density $\mu$, then only the topologically Euclidean solution (4.4), the quasi-regular solution (4.27) and the exceptional wormhole solution (5.15) remain. In these cases incoming test particles are always reflected back, so that total or partial absorption of the incident beam would signal that the matter making up the cylinder is exotic.

After this work was completed, we came across an article by Khorrami and Mansouri [19]. The hollow cylinder considered by these authors is nonrotating $(\beta=0)$, but may collapse or expand. Assuming the exterior metric to be the static cosmic string metric, and choosing the interior metric to be the topologically Euclidean metric (4.4), the authors of [19] recover our relation (4.1) $\tau_{z}=\mu+\tau_{\varphi}$, and find that the static solution corresponds to $\tau_{\varphi}=0$ (which results from our eq. (4.7) with $\beta=0$ ).

\footnotetext{
${ }^{3}$ The purported demonstration of eq. (31) is flawed.
} 


\section{Acknowledgement}

Part of this work was carried out while G. C. was at the Institut Non Linéaire de Nice (INLN). I. Z. wishes to thank the INLN and Professor F. Rocca (Université de Nice) for the kind hospitality afforded during part of this work. 


\section{References}

[1] A. Vilenkin, Phys. Rev. D 23, 582 (1981).

[2] J. Gott, Astrophys. J. 288, 422 (1985);

W.A. Hiscock, Phys. Rev. D 31, 3288 (1985);

B. Linet, Gen. Rel. Grav. 17, 1109 (1985).

[3] E. Frehland, Comm. Math. Phys. 26, 307 (1972).

[4] G. Clément, Ann. Phys. (N.Y.) 201, 241, (1990).

[5] W. Israel, Nuovo Cim. 44B, 1 (1966); 48B, 463 (1967).

[6] L.D. Landau and E.M. Lifchitz, The Classical Theory of Fields (Pergamon, New York, 1975).

[7] H. Davies and T.A. Caplan, Proc. Camb. Phil. Soc. 69, 325 (1971);

E. Frehland, Comm. Math. Phys. 23, 127 (1971).

[8] D. Kramer, H. Stefani, M. MacCallum and E. Herlt, Exact Solutions of Einstein's Field Equations (VEB Deutscher Verlag der Wissenschaften, Berlin, 1980).

[9] E. Kasner, Amer. J. Math. 43, 217 (1921).

[10] A. Harvey, Gen. Rel. Grav. 22, 1433 (1990), and references therein.

[11] G. Clément, Gen. Rel. Grav. 18, 861 (1986).

[12] S. Deser, R. Jackiw and G. 't Hooft, Ann. Phys. (N.Y.) 152, 220 (1984).

[13] W. Rindler, Essential Relativity (Springer Verlag, New York, 1977).

[14] G. Clément, preprint GCR-94/02/01 (1994).

[15] G.F.R. Ellis and B.G. Schmidt, Gen. Rel. Grav. 8, 915 (1977).

[16] S.J. Hugues, D.J. Mc Manus and M.A. Vandyck, Phys. Rev. D 47, 468 (1993);

D.J. Mc Manus and M.A. Vandyck, Phys. Rev. D 47, 1491 (1993). 
[17] I. Zouzou, Magister thesis (Constantine, 1994).

[18] M. Visser, Phys. Rev. D 39, 3182 (1989); Nucl. Phys. B 328, 203 (1989).

[19] M. Khorrami and R. Mansouri, J. Math. Phys. 35, 951 (1994). 
Figure 1: Penrose diagram for the black-hole space-time generated by two mirror-symmetric matter cylinders (heavy curved lines). The exterior regions are truncated conical space-times, while the interior region is a truncated Rindler cylinder. 
This figure "fig1-1.png" is available in "png" format from: http://arxiv.org/ps/gr-qc/9405074v1 